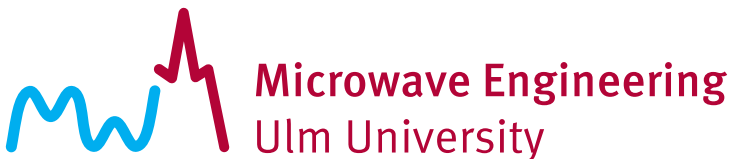

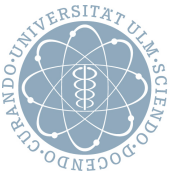

\section{Digital Beamforming to Mitigate Automotive Radar Interference}

\author{
Jonathan Bechter, Karim Eid, Fabian Roos, and Christian Waldschmidt
}

(C) 2016 IEEE. Personal use of this material is permitted. Permission from IEEE must be obtained for all other uses, in any current or future media, including reprinting/republishing this material for advertising or promotional purposes, creating new collective works, for resale or redistribution to servers or lists, or reuse of any copyrighted component of this work in other works. 


\title{
Digital Beamforming to Mitigate Automotive Radar Interference
}

\author{
Jonathan Bechter, Karim Eid, Fabian Roos, and Christian Waldschmidt \\ Institute of Microwave Engineering, Ulm University, Germany \\ Email: jonathan.bechter@uni-ulm.de
}

\begin{abstract}
Interference between automotive radars decreases the sensors' detection capabilities. It is possible to use digital beamforming (DBF) in multi-antenna systems to reduce the power received from certain directions of arrival (DoA). If digital beamforming is used to mitigate the effect of an interferer, it is shown that it is not sufficient to cancel the DoA of an interferer alone, if an I-Q mixer is not present. Additionally, a second DoA must be blinded out. A DBF system which performs this task is presented. Experimental and simulated results support the mathematical derivation and show possible improvements with DBF.
\end{abstract}

\section{INTRODUCTION}

With the widespread usage of radar in driver assistance systems and a run towards autonomous driving, the density of radar sensors operating simultaneously on the road is increasing. However, the available bandwidth is limited. This increases the probability that sensors disturb each other, leading to unwanted interference. Automotive radars typically use modulation schemes with linear frequency ramps, like FMCW or chirp sequence modulation. Therefore, interference of such systems is considered in the following.

Interference from other sensors can decrease the distance between target peaks and noise floor [1], [2]. To prevent a reduction of the signal to noise ratio, a possible countermeasure is the usage of digital beamforming (DBF) to remove an interferer's influence. DBF to cancel out interference between automotive radars has already been addressed in [3]. In [3], the direction of an interferer is estimated in time domain and afterwards canceled using an adaptive beamformer. Here, we will compare an estimation of the interferer's direction in time and frequency domain. This direction is then notched out. An adaptive beamformer is not used here, as only a single interferer with known location shall be removed. It will be shown that it is not sufficient to just notch out the one direction where the interferer is located, but that its power is split over two directions in space domain. This effect occurs if no I-Q mixer is used, and its consequences will be investigated in the next sections.

The paper describes the occurrence of interference between automotive radars, followed by the DoA (direction of arrival) estimation of an interfering signal. Then, the mathematical model to cancel the interference with a beamformer will be derived. Results from simulations and measurements support the theoretical derivations and indicate the possible performance.

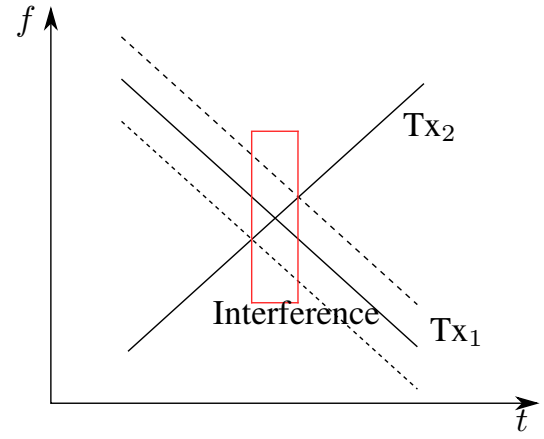

Fig. 1. Two radar sensors transmit signals $\mathrm{Tx}_{1}$ and $\mathrm{Tx}_{2}$ with an overlap in time and frequency, leading to unwanted interference. The interference is limited in time because of the receiver bandwidth. This is indicated by the dotted lines for the signal $\mathrm{Tx}_{1}$.

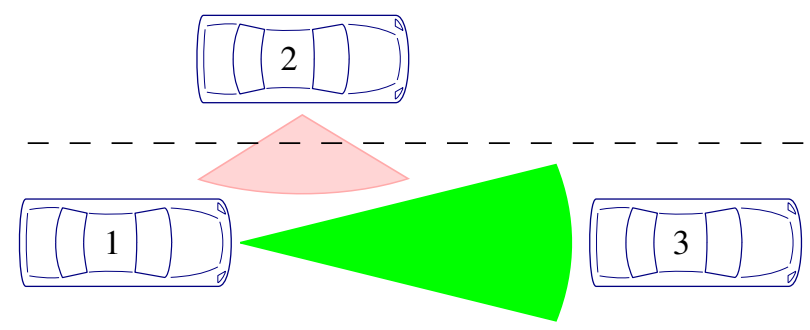

Fig. 2. In this scenario, the sensor of car 2 causes interference from a short distance with its blind spot sensor while overtaking car 1 .

\section{Setup of the Digital Beamformer}

\section{A. Problem Description and Background Knowledge}

A scenario is considered in which two radar sensors transmit linear frequency ramps as in Fig. 1. As the sensors transmit at the same time in overlapping frequency bands, the frequency ramps cross each other. When a ramp falls within the receiver bandwidth of the other sensor (indicated by the dotted line), time limited interference occurs. For the non-parallel ramps, this results in an increase of the overall noise floor in the spectrum [4]. This could happen in a setup as shown in Fig. 2, in which the car on the left lane passes the car on the right. A blind spot sensor can cause interference from close proximity.

As digital beamforming is used to mitigate this effect, its basic working principle is described. Between two elements (e.g. the $i$-th and $(i+1)$-th) of a receiving array, each signal will be phase-shifted by $\Delta \varphi_{i+1, i}$ according to its angle of 
incidence $\vartheta$ :

$$
\Delta \varphi_{i+1, i}=\varphi_{i+1}(t)-\varphi_{i}(t)=2 \pi \frac{d}{\lambda} \sin \vartheta
$$

The distance $d$ between the two receiving elements with signal phases $\varphi_{i}(t)$ and $\varphi_{i+1}(t)$ and the wavelength $\lambda$ of the signal are known. The input signals $s_{i}(t)$ of the different receiving elements are multiplied by weighting factors $w_{i}$. The sum of these products is the beamformer output $s(t)$ [5]:

$$
s(t)=\sum_{i} s_{i}(t) \cdot w_{i} .
$$

Because of the Fourier Transform's linearity, the operation in (2) can also be applied in the frequency domain.

\section{B. Detection of the Direction of Arrival of an Interferer}

In this section, it is shown why the power of an interferer is split over two different incidence angles. Afterwards, the DoA estimation of an interferer is explained.

In a chirp sequence or FMCW modulated radar system without an I-Q mixer, each target in the radar channel yields a baseband signal of the form

$$
\cos (x)=\frac{1}{2}\left(e^{j x}+e^{-j x}\right) .
$$

Signal processing is done by calculating a Fourier Transform. Each exponential function in (3) results in one peak in the frequency spectrum. Those two peaks are symmetric, see Fig. 3. Only one half of the spectrum represents positive distances, so the ambiguity can be solved.

If multiple receiving channels are present, the DoA of a target can be determined by applying an estimation algorithm on that peak, which corresponds to the positive distance. If the estimation is applied on the other peak, it will result in the same DoA with opposite sign, as the two peaks are complex conjugate. If a DoA estimation is performed on the time domain data, this results in both DoAs, as the real valued time domain signals contains the two complex conjugate exponential functions, see (3).

The DoA of the interferer is estimated in time or frequency domain. For estimation in time domain, the interference must be detected, e.g. with a power detector, as the interference affects only a part of the signal. Afterwards, a DoA estimator is applied, as in [3]. Such an estimation is also done for an interference free part of the time domain signal. The difference

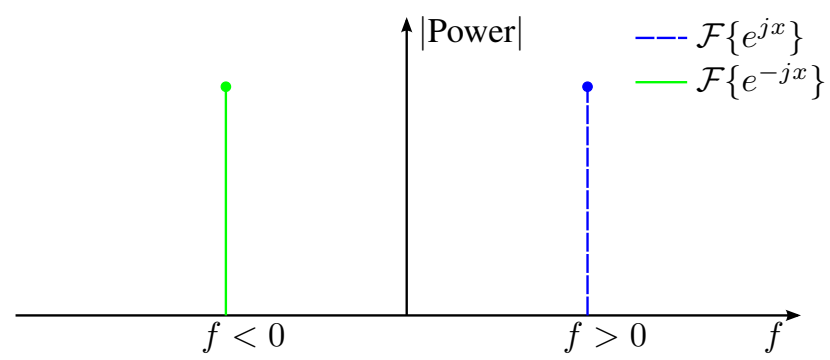

Fig. 3. The constant frequency of the baseband signal of a radar target leads to two peaks separated in the positive and negative half of the spectrum. of both estimations leads to the DoA of the interferer, but there will be two solutions.

As mentioned before, the power of the interference is spread over the whole frequency spectrum. Thus, the DoA estimation in the spectrum can be done for any frequency bins. For a signal from a target, the DoA in the spectrum is unambiguous. But, for an interfering signal the DoA estimation in the spectrum yields two angles of arrival with opposite signs. This behavior can be explained by the properties of the Fourier Transform. In time domain, the interfering signal $s(t)$ is described generally as

$$
\begin{aligned}
s\left(t-T_{0}\right) & \left.\propto \cos \left(a\left(t-T_{0}\right)^{2}+b\right)\right) \\
& =\frac{1}{2}\left(e^{j\left(a\left(t-T_{0}\right)^{2}+b\right)}+e^{-j\left(a\left(t-T_{0}\right)^{2}+b\right)}\right) .
\end{aligned}
$$

The coefficients $a$ and $b$ are here arbitrary variables, $T_{0}$ indicates the point in time when the interference is occurring. A more detailed description of the equation is given e.g. in [2]. Information on the interference's DoA is included in the timeindependent variable $b$ which appears with opposite sign in the two exponential functions. For the Fourier Transform of (4), it can be stated that

$$
s\left(t-T_{0}\right) \bullet \multimap S(f) \cdot e^{-j 2 \pi f T_{0}},
$$

with $S(f)=\mathcal{F}\{s(t)\}$. In (3), each exponential function led to a single peak on one side of the spectrum for target responses. For an interference, this is not the case, compare Fig. 4. Because of

$$
e^{j\left(a(t)^{2}+b\right)}=e^{j\left(a(-t)^{2}+b\right)},
$$

such an exponential function is even in time domain. According to the common relationships

$$
\begin{aligned}
& \text { real valued, even } \quad \bullet \quad \text { real valued, even } \\
& \text { imaginary, even } \bullet \longrightarrow \quad \text { imaginary, even }
\end{aligned}
$$

its Fourier Transform is also an even function in frequency domain. As the Fourier Transform of each single exponential function in (4) is present in both the negative and the positive side of the spectrum, also their angular information is present there. Therefore, the interference spreads its power onto two angles in each bin of the spectrum. This leads to the problem that a beamformer must cancel both of those angles in order to completely remove the interference induced noise floor. This

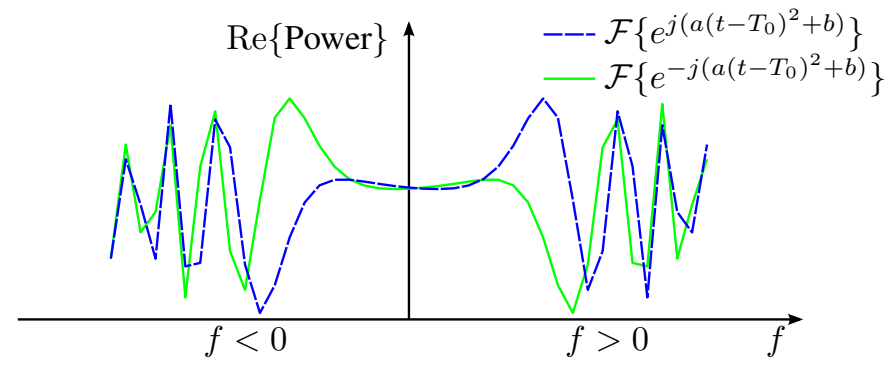

Fig. 4. Each exponential function of the interfering signal has components in both sides of the spectrum. For better visibility, only the real parts are shown. 
problem can be avoided by using an I-Q mixer, as this kind of mixer removes one of the exponential functions in (4).

\section{Removing Undesired Signals}

A system with four receiving channels is considered to demonstrate how an interference can be removed. The method is not limited to four receiving channels, but in the measurement setup the same amount is available. In the frequency spectrum, an interfering signal on channel $i$ is modeled as

$$
S_{\text {int }, i}(f)=S_{\text {int }}(f) \cdot\left(e^{j \varphi_{i}}+e^{-j \varphi_{i}}\right) .
$$

The subscript int is related to the interference, $S_{\text {int }}(f)$ is its frequency response common on all channels. According to (1), there is a different phase $\varphi_{i}$ on each channel. Two exponential functions are summed up in (7), which means the interfering signal appears at two angles with opposite sign. When the direction of the interference source is determined as shown above, then the phase differences between the channels can be calculated. The interfering signal at any frequency bin is present as a sum of two complex vectors, each varying over the channels by $\pm \Delta \varphi_{i}$. The weights of the beamformer are chosen in a way that the interfering signal is canceled in two channels:

$$
w_{1} \cdot S_{\mathrm{int}, 1}(f)+w_{2} \cdot S_{\mathrm{int}, 2}(f)=0 .
$$

This leads to

$$
\begin{aligned}
& w_{1}=1 \\
& w_{2}=e^{-j\left(\varphi_{2}-\varphi_{1}+\pi\right)} .
\end{aligned}
$$

Accordingly, for $w_{3}$ and $w_{4}$ follows

$$
\begin{aligned}
& w_{3}=1 \\
& w_{4}=e^{-j\left(\varphi_{4}-\varphi_{3}+\pi\right)} .
\end{aligned}
$$

This operation removes the interference related to the phase shift $+\Delta \varphi_{i}$. To completely remove the interference, one pair of weights has to be adjusted to address the interference with phase shift $-\Delta \varphi_{i}$. So, $w_{3}$ and $w_{4}$ are multiplied with a complex value $a$ such that

$$
\begin{array}{r}
w_{1} \cdot e^{j 0}+w_{2} \cdot e^{-j \Delta \varphi_{21}}+a \cdot\left(w_{3} \cdot e^{-j \Delta \varphi_{31}}+w_{4} \cdot e^{-j \Delta \varphi_{41}}\right) \\
\quad=1+w_{2} \cdot e^{-j \Delta \varphi_{21}}+a \cdot\left(e^{-j \Delta \varphi_{31}}+w_{4} \cdot e^{-j \Delta \varphi_{41}}\right)=0 .
\end{array}
$$

This leads to

$$
a=-\frac{1+w_{2} \cdot e^{-j \Delta \varphi_{21}}}{e^{-j \Delta \varphi_{31}}+w_{4} \cdot e^{-j \Delta \varphi_{41}}} .
$$

A digital beamformer with the weights $w_{1}, w_{2}, \hat{w}_{3}=a w_{3}$ and $\hat{w}_{4}=a w_{4}$ will cancel the interference completely, while using the weights (9) - (12) removes only half of the interference power. When the interference is removed completely, no more degrees of freedom for choosing the weights are left. This means, that it is not possible to optimize directions of interest with the beamformer anymore. It will be seen in the measurement section, that the target signals will be arbitrarily amplified or damped. To additionally amplify signals from certain directions, an array with more elements is required.

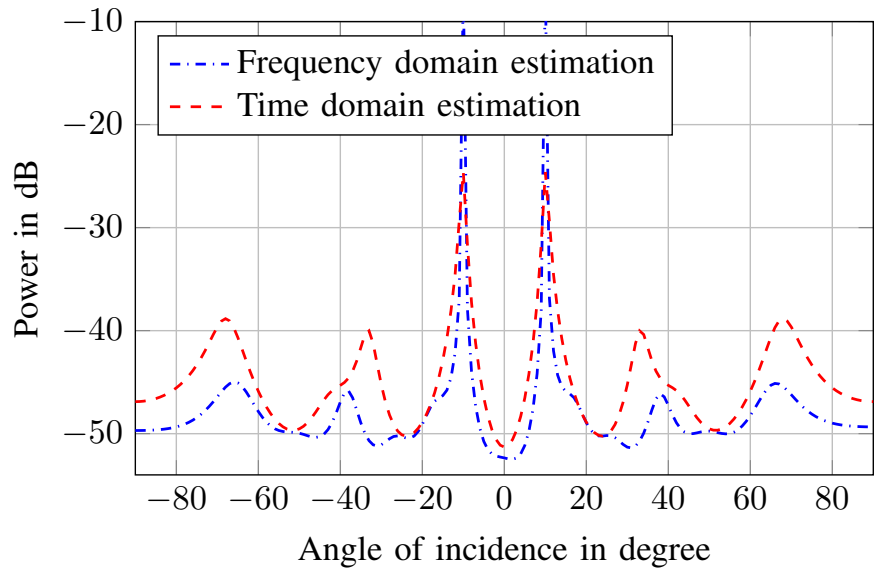

Fig. 5. A DoA estimation is performed on samples affected by interference in time domain and on bins without strong peaks from targets in frequency domain. In both cases, the interference is estimated at an angle of $\pm 10^{\circ}$. Its power is split onto two incident angles. As the array got high side lobes, additional peaks with lower amplitude occur.

\section{Simulative Verification}

The receiving array consists of four non-uniformly spaced elements with antenna distances larger than $\lambda / 2$, so the array has high side lobes. The radar parameters used for the sensor affected by interference are a center frequency of $76 \mathrm{GHz}$ and a bandwidth of $800 \mathrm{MHz}$. Interference is caused by a CW signal at $76 \mathrm{GHz}$. The interference source is located at an angle of $-10^{\circ}$ and a distance of $6 \mathrm{~m}$. Additional targets are at $6^{\circ}, 4.6 \mathrm{~m}$, and $12^{\circ}, 3.4 \mathrm{~m}$.

To estimate the incidence angle of the interference, the Capon Algorithm [5] is used. In Fig. 5 the DoA estimations of the interferer are shown for both the time domain samples and the frequency spectrum. When bins including strong peaks of targets are excluded from the frequency domain estimation, it is assured that the result of the estimation belongs to the interference signal. The interference is estimated at the angles $\pm 10^{\circ}$ in both cases. This matches with the statement that the interference appears under two angles of incidence. In the figure, the DoAs of the targets cannot be seen properly, although they should be present in the time domain estimation. The reason is, that the interference with the much higher amplitude dominates the estimation.

With the two DoAs known, the interference is removed from the spectrum using the weights $w_{1}, w_{2}, \hat{w}_{3}$ and $\hat{w}_{4}$ according to (9) - (14). In the simulation, the interference induced noise floor was reduced by approximately $40 \mathrm{~dB}$.

\section{MeAsurement}

Measurements were taken in an anechoic chamber with targets and an interference source at the same locations as in the simulations. The victim sensor is a chirp sequence modulated automotive radar, also with parameters according to the simulation. As an interference source, a $\mathrm{CW}$ signal at $76 \mathrm{GHz}$ is fed to a horn antenna. The victim was rotated on a 


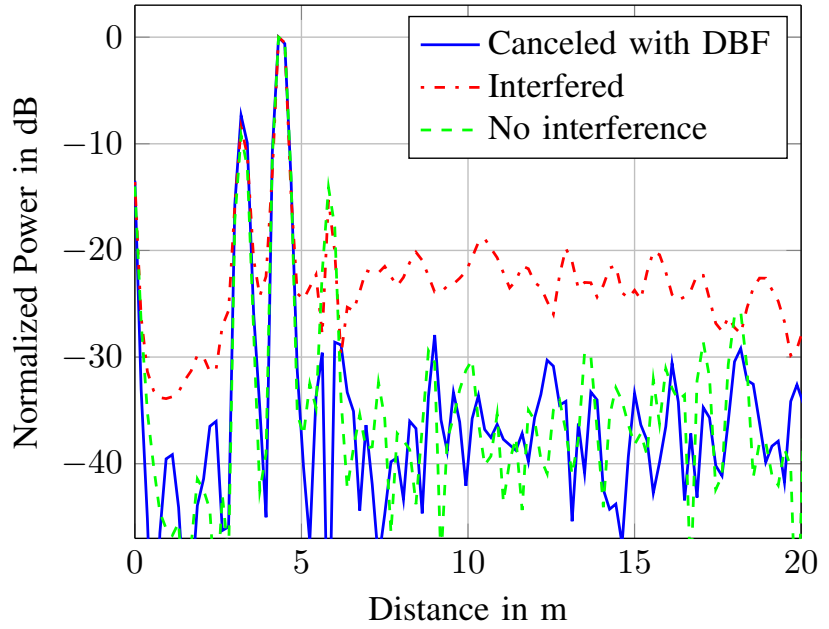

Fig. 6. Measurement showing the performance of the described DBF system. The signal with interference present has an average noise floor of $-26.7 \mathrm{~dB}$. Using DBF reduces the noise floor to $-37.5 \mathrm{~dB}$. Without interference, the average noise floor is $-38.4 \mathrm{~dB}$. In the figure, only a snapshot of the spectrum is shown.

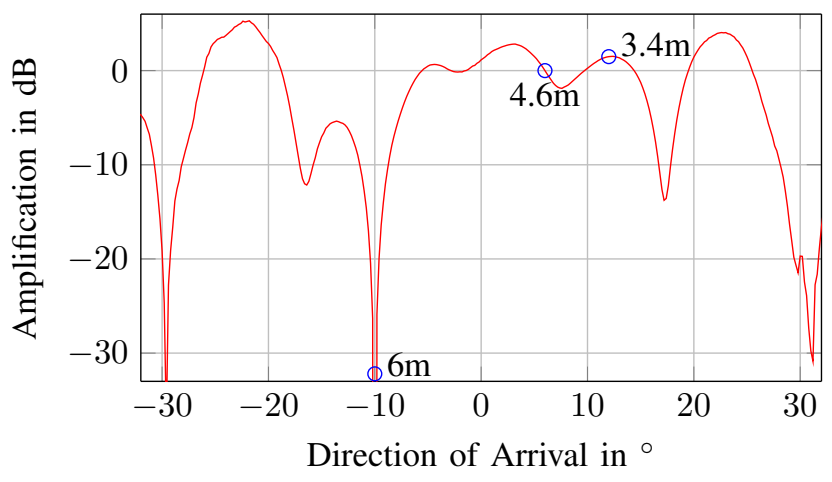

Fig. 7. The red curve shows how the beamformer fortifies and damps signals depending on their DoAs, while canceling an interferer at $-10^{\circ}$. The blue dots indicate the locations of the targets in Fig. 6.

turntable, so it was possible to create scenarios with different angles towards targets and interferer.

With the interferer and two targets at positions according to the simulations, beamforming is used to cancel the interference with the weights derived above. The beamformer output is shown in Fig. 6 (blue curve), all curves are normalized to the power of the strongest target (corner reflector in $4.6 \mathrm{~m}$ ). It can be seen that the interference induced noise floor (red curve) is removed nearly completely compared to the same uninterfered signal (green curve). Obviously, the target related to the interferer (distance $6 \mathrm{~m}$ ) is reduced. Fig. 7 shows, how the beamformer is amplifying or damping signals depending on their DoA with the chosen weights. The target at $6^{\circ}$ is slightly less amplified than the target at $12^{\circ}$. At $-10^{\circ}$ is a notch for the interferer, but there is no second notch at $+10^{\circ}$ for its second incident angle. The calibration required for real DBF systems transforms this power to another incident angle, which is then canceled by the beamformer.

By rotating the victim radar, different angles towards the
TABLE I

Average Noise Floor Measured in Different Scenarios

\begin{tabular}{|c||c|c|c|}
\hline Interferer Angle & $0^{\circ}$ & $-5^{\circ}$ & $-10^{\circ}$ \\
\hline Interfered, no DBF & $-16 \mathrm{~dB}$ & $-22.9 \mathrm{~dB}$ & $-26.7 \mathrm{~dB}$ \\
\hline One DoA canceled & $-20 \mathrm{~dB}$ & $-27.8 \mathrm{~dB}$ & $-33.5 \mathrm{~dB}$ \\
\hline Two DoAs canceled & $-29.9 \mathrm{~dB}$ & $-37.7 \mathrm{~dB}$ & $-37.5 \mathrm{~dB}$ \\
\hline Interference free, no DBF & $-31.1 \mathrm{~dB}$ & $-35.6 \mathrm{~dB}$ & $-38.4 \mathrm{~dB}$ \\
\hline
\end{tabular}

interferer are set. For multiple scenarios, the average noise floor is calculated. The region of the spectrum containing the three targets is not considered for these calculations. Table I shows the average noise floors calculated with the interferer under different DoAs. The noise floor when canceling only one incidence angle of the interferer (by setting $a=1$ in (14)) is compared to the noise floor when two DoAs are canceled. The comparison of the different noise floors with the interfered and interference free signals shows, that removing only the interferer's DoA is not sufficient to evade its effects. It is required to additionally cancel the second DoA according to the interferer. Also, it is possible to reach a better SNR as in the interference free case with the gain of the beamformer. The data was again normalized to the power of the strongest target as in Fig. 6.

\section{CONCLUSION}

When using digital beamforming to remove interference between automotive radars, it is not sufficient to remove only the direction of an interferer if no I-Q mixer is used. It was proven that the interferer will split its power onto two angles of incidence. In an ideal system, the second angle is exactly that of the interferer just with the opposite sign. By estimating an interferer's DoA, the required weights to cancel its influence with a digital beamformer can be determined. In measurements, an interference induced noise floor of up to $15 \mathrm{~dB}$ could be removed from the spectrum. However, the SNR of targets can also decrease. In simulations, it was possible to achieve an improvement of the SNR of $40 \mathrm{~dB}$.

\section{REFERENCES}

[1] G. M. Brooker, "Mutual Interference of Millimeter-Wave Radar Systems," IEEE Transactions on Electromagnetic Compatibility, vol. 49, no. 1, pp. 170-181, Feb. 2007.

[2] T. Schipper, S. Prophet, M. Harter, L. Zwirello, and T. Zwick, "Simulative Prediction of the Interference Potential Between Radars in Common Road Scenarios," IEEE Transactions on Electromagnetic Compatibility, vol. 57, no. 3, pp. 322-328, Jun. 2015.

[3] C. Fischer, H. Blocher, J. Dickmann, and W. Menzel, "Robust Detection and Mitigation of Mutual Interference in Automotive Radar," in 16th International Radar Symposium (IRS), Jun. 2015, pp. 143-148.

[4] M. Goppelt, H.-L. Blöcher, and W. Menzel, "Automotive radar investigation of mutual interference mechanisms," Advances in Radio Science, vol. 8, pp. 55-60, 2010. [Online]. Available: http://www.advradio-sci.net/8/55/2010/

[5] S. Haykin and K. J. Ray Liu, Handbook on array processing and sensor networks. Hoboken, N.J.: Wiley, 2009. [Online]. Available: http://proquest.tech.safaribooksonline.de/9780470371763 